\section{Twenty- or 24F-diameter dilation prevents severe bleeding associated with 27F- diameter dilation for direct percutaneous endoscopic gastrostomy tube placement}

\author{
Akira Horiuchi, ${ }^{1}$ Yoshiko Nakayama, ${ }^{1,2}$ \\ Naoki Tanaka ${ }^{1,3}$ \\ 'Digestive Disease Center, Showa Inan \\ General Hospital, Komagane; \\ 2Department of Pediatrics, Shinshu \\ University School of Medicine; \\ ${ }^{3}$ Department of Metabolic Regulation, \\ Shinshu University Graduate School of \\ Medicine, Matsumoto, Japan
}

\section{Abstract}

Severe bleeding sometimes occurs after percutaneous endoscopic gastrostomy (PEG) placement using a modified introducer method (direct method). We hypothesized that using a smaller diameter dilator rather than the $27 \mathrm{~F}$ dilator contained in the commercial kit would reduce the incidence of this complication. The aim of this study was to compare procedurerelated complications with the use of $27 \mathrm{~F}$ dilators and 20F/24F dilators. Consecutive patients who underwent the direct method for PEG placement were studied. The $27 \mathrm{~F}$ dilator was used from January 2007 to July 2008 (27F dilator group) and the $20 \mathrm{~F}$ and $24 \mathrm{~F}$ dilators were used from August 2008 to December 2009 (20F/24F dilator group). The success rates, procedure times, and procedure-related complications within 30 days of the procedure were evaluated retrospectively in 136 patients: 66 in the $27 \mathrm{~F}$ dilator group and 70 in the $20 \mathrm{~F} / 24 \mathrm{~F}$ dilator group. The success rates and mean procedure times of the groups were similar. Severe bleeding occurred in five patients in the $27 \mathrm{~F}$ dilator group versus none in the smaller dilator group $(\mathrm{P}=0.025)$. Complications such as peristomal infection, peritonitis, and respiratory depression did not occur in either group. There was no significant difference in 30-day mortality between the groups ( 2 vs. $1, \mathrm{P}=0.61$ ). We concluded that the direct method using 20F/24F dilators has less procedure-related severe bleeding than that using the $27 \mathrm{~F}$ dilator contained in the commercial kit of $24 \mathrm{~F}$ button-bumper-type devices.

\section{Introduction}

The pull/push and introducer methods are established as standard techniques for percu- taneous endoscopic gastrostomy (PEG) placement. In the pul/push method, the feeding tube is introduced through the mouth and thus requires a reasonably patent esophagus. In contrast, balloon-type catheter feed tubes can be inserted directly into the stomach through the abdominal wall using an introducer method. Recently, we have reported a modified introducer method (direct method) that includes fixation of the gastric wall to the abdominal wall using a gastropexy device and then dilation of the hole using a dilator, which allows direct placement of a larger buttonbumper-type catheter device and reduces the incidence of catheter changes, compared with a 20F catheter in the standard pull method. ${ }^{1}$

The major complications of the standard pull method, which requires an esophageal lumen sufficient to pass a standard endoscope ${ }^{2,3}$ include peristomal wound infections presumably resulting from contamination of the gastrostomy catheter as it passes through the oral cavity, ${ }^{4,5}$ and tumor implantation at the PEG site. ${ }^{6}$ The disadvantages of the introducer method are that only small diameter balloontype catheters are available and the requirement of frequent catheter changes when longterm tube feeding is needed. ${ }^{7,8}$ In addition, procedure-related severe bleeding associated with the direct method has been reported. ${ }^{1} \mathrm{We}$ hypothesized that bleeding was associated with the use of the $27 \mathrm{~F}$ dilator in the commercial kit. The aim of our study was to compare complications associated with the use of $27 \mathrm{~F}$ dilators and $20 \mathrm{~F} / 24 \mathrm{~F}$ dilators during the direct method of PEG placement.

\section{Materials and Methods}

\section{Patients}

Consecutive patients, over 18 years of age, who were referred for the placement of a button-bumper-type catheter and who underwent initial PEG placement using a direct method for dysphagia between January 2007 and December 2009 were screened for the study. The exclusion criteria included: signs of acute infection, prior gastric surgery, or having been assigned to the American Society of Anesthesiologists class IV. Coagulation status, such as PT, PTT, INR, and the platelet levels during the procedure were within normal limits in all patients. In addition, antiplatelet agents and anticoagulants were not used in any patients. Written informed consent was obtained before the procedure. For patients unable to give consent, consent was obtained from family members. This retrospective study was approved by the ethics committee at our hospital.
Correspondence: Akira Horiuchi, Digestive Disease Center, Showa Inan General Hospital, 3230 Akaho, Komagane 399-4191, Japan.

E-mail: horiuchi.akira@sihp.jp

Key words: percutaneous endoscopic gastrostomy, direct method, modified introducer method, bleeding.

Contributions: AH, YN, acquisition of data; $\mathrm{AH}$, $\mathrm{NT}$, analysis and interpretation of data; $\mathrm{AH}$, drafting of the manuscript; YN, NT, critical revision of the manuscript.

Conflict of interest: the authors report no conflicts of interest.

Received for publication: 29 March 2010.

Revision received: 18 May 2010.

Accepted for publication: 23 May 2010.

This work is licensed under a Creative Commons Attribution 3.0 License (by-nc 3.0).

(C) Copyright A. Horiuchi et al., 2010

Licensee PAGEPress, Italy

Gastroenterology Insights 2010; 2:e8

doi:10.4081/gi.2010.e8

\section{Percutaneous endoscopic}

\section{gastrostomy device}

The bumper-button-type PEG tube (Ideal button, Olympus, Tokyo, Japan) was a $24 \mathrm{~F}$ dilator and available in lengths of 2.0, 2.5, 3.0, 3.5, 4.0 , and $4.5 \mathrm{~cm}$. The obturator supplied with the PEG tube fits into the button to extend the PEG tube to minimize stomal damage during either insertion or withdrawal. The PEG tube can also be placed over a guidewire $(0.035$ in, $80 \mathrm{~cm})$. The commercial kit contained an obturator, a 27F dilator, a guidewire, a measuring device, and a double-lumen gastropexy device.

\section{The direct method}

The procedure was written in detail in our previous report. ${ }^{1}$ The stomach was distended and the abdominal wall was trans-illuminated; the site for the PEG placement was anesthetized with an injection of a few milliliters of $1 \%$ lidocaine. The site was punctured with the gastropexy device (Olympus, Tokyo, Japan) under aseptic conditions. ${ }^{7-9}$ After both tips of the gastropexy device were confirmed to be intragastric, a wire snare loop was inserted through the first channel of the gastropexy device and the snare loop was opened. Then a suture was inserted through the second channel of the gastropexy device. Once in the stomach, the suture was grasped with the snare loop, the snare loop with the suture was withdrawn, and the suture was fixed. Then the gastropexy device was removed and the gas- 
tropexy suture was tied. In the same fashion, a second gastropexy suture was applied so that there was a gap of approximately $2 \mathrm{~cm}$ between each suture knot. An incision was made in the center between the two gastropexy sutures and an 18-gauge needle was introduced into the stomach. A guidewire was introduced through the needle and the skin incision was dilated by passing a $27 \mathrm{~F}$ dilator, contained in the commercial kit, or two kinds of dilators typically used for urology (20F and 24F) (Cook Japan, Tokyo, Japan), percutaneously into the stomach over the guidewire during endoscopic observation. After the dilator was removed, a measuring device was inserted over the guidewire to determine the length of the button, followed by insertion of the PEG tube using an obturator. The length of the PEG button was selected based on the measurement that included the thickness of the anterior gastric wall, the abdominal wall, and a 1-cm addition. The gastropexy sutures were removed after 14 days.

\section{Study design}

From January 2007 to July 2008, the 27F dilator contained in the commercial kit was used for the dilation. From August 2008 to December 2009, the 20F/24F dilators were used. The procedure was the same in both groups except for the dilators. All procedures, irrespective of the method, were performed by two endoscopists who had each done more than 50 such procedures before this study. Procedures were carried out under local anesthesia and intravenous administration of propofol (20-60 mg per procedure). Prophylactic antibiotics (2 g per day of intravenous ceftriaxone) were used in all patients shortly before and for three days after the procedure. Enteral nutrition was initiated in all patients on the third day after the procedure.

The success rate, duration, and complications of both procedures were compared retrospectively in the two groups. We recorded the presence of complications including bleeding, peristomal infection, peritonitis, and respiratory depression. Bleeding was defined as the decrease of more than $1 \mathrm{~g} / \mathrm{dL}$ of hemoglobin and/or the requirement for blood transfusion. The peristomal infection rate was determined and the peristomal site was evaluated once a day in the time interval between the date of placement and the date of discharge. Peristomal infection was defined as having at least two of the following: peristomal erythema, indurations, and purulent discharge, irrespective of size or degree. ${ }^{5}$ Peristomal wound cultures were obtained from any purulent discharge at the catheter exit site. Respiratory depression was defined as the continued need for oxygen supply after the procedure. Mortality within the 30th day after the procedure was reported by the health care

Table 1. Demographic and baseline characteristics of 136 patients.

$\begin{array}{lccc}\text { Groulp } & \begin{array}{c}\text { 27F dilator } \\ (\mathrm{N}=66)\end{array} & \begin{array}{c}20 \mathrm{~F} / 24 \mathrm{~F} \text { dilators } \\ (\mathrm{N}=70)\end{array} & \mathrm{P} \\ \text { Gender (male/female) } & 40 / 26 & 43 / 27 & 0.92 \\ \text { Age (years) (mean } \pm \mathrm{SD}) & 81.5 \pm 5 & 82.1 \pm 8 & 0.72 \\ \text { Indication for PEG: CVA/CNSD/tumor } & 55 / 10 / 1 & 50 / 14 / 6 & 0.58 \\ \text { Number of previous feedings: PO/NGT/PEN } & 14 / 40 / 12 & 15 / 42 / 13 & 0.99\end{array}$

PEG, percutaneous endoscopic gastrostomy; CVA, cerebrovascular accident; CNSD, central nervous system disorders; PO, per oral feeding; NGT, nasogastric tube feeding; PEN, parenteral nutrition. Values are numbers of patients except for age. 27F-dilator group: January 2007 to July 2008 (19 months); 20F/24F-dilator group: August 2008 to December 2009 (17 months).

Table 2. Comparison of outcomes and complications (30 days post-procedures) of using 27F dilators and 20F/24F dilators during the direct method for PEG placement.

\begin{tabular}{lccc} 
Group & $\begin{array}{c}27 \mathrm{~F} \text { dilator } \\
(\mathrm{N}=66)\end{array}$ & $\begin{array}{c}20 \mathrm{~F} / 24 \mathrm{~F} \text { dilators } \\
(\mathrm{N}=70)\end{array}$ & $\mathrm{P}$ \\
Successful procedure & $66(100)$ & $70(100)$ & \\
Procedure time (min) (mean $\pm \mathrm{SD})$ & $10 \pm 3.1$ & $9.5 \pm 3.6$ & 0.78 \\
Complications & 0 & 0 & \\
$\quad$ Peristomal infection & $5(7.6)$ & 0 & 0.025 \\
Bleeding & 0 & 0 & \\
Peritonitis & 0 & 0 & 0.61 \\
Respiratory depression & $2(3.0)$ & $1(1.4)$ & \\
30-day mortality & & & \\
\hline
\end{tabular}

PEG, percutaneous endoscopic gastrostomy. Values are numbers (percentages) of patients except for procedure time. Bleeding was defined as a decrease of more than $1 \mathrm{~g} / \mathrm{dL}$ of hemoglobin and/or requirement for blood transfusion.

providers for the patients. Other parameters recorded included demographic data (i.e. age, sex, previous feeding status, and indications for the procedure).

\section{Statistical analysis}

Statistical tests to compare the results for the two groups were as follows: the Chi-square test, with Yates' correction for continuity where appropriate, was used for comparison of cat egorical data; Fisher's exact test was used when the numbers were small; for parametric data, the Student's t-test was used when two means were compared. Differences were considered significant if the P value was less than 0.05 .

\section{Results}

A total of 136 patients were studied retrospectively. There were no significant differences between the two groups with respect to clinical baseline parameters including age, gender, indications, and mode of previous feeding (Table 1). The subjects were elderly patients with a relatively poor nutrition state. The success rates of the groups were identical (100\%) and the procedure times were similar $(10 \pm 3.1 \mathrm{~min}, 9.5 \pm 3.6 \mathrm{~min}, \mathrm{P}=0.78)$. Five patients in the 27F-dilator group had bleeding from the insertion site compared to none in the 20F/24F-dilator group ( $\mathrm{P}=0.025)$ (Table 2). Hematemesis occurred at four to six hours after PEG placement. The bleeding appeared to originate from the gastrostomy sites and was associated with a decrease of $1-3 \mathrm{~g} / \mathrm{dL}$ of hemoglobin. Other complications such as peristomal infection, peritonitis, and respiratory depression did not occur in either group. Two patients in the 27F-dilator group and one patient in the 20F/24F-dilator group died from aspiration pneumonia caused by vomiting after PEG feeding within 30 days of PEG placement.

\section{Discussion}

We previously reported that the advantages of the direct method were: i) initial placement of a button-bumper-type catheter of relatively large size making fluid delivery more efficient and having a longer life span than that of balloon type catheters; ii) transnasal endoscopy can be used to guide PEG placement; iii) fewer peristomal wound infections occurred compared with that of the standard pull method; iv) the avoidance of the potential for tumor seeding that can occur when traditional PEG placement is performed in patients with neck and esophageal cancers. ${ }^{1}$ This study demonstrated again that peristomal infection did not occur and that the use of the $27 \mathrm{~F}$ dilator was associated with post-procedure bleeding. We also showed that use of smaller dilators prevented procedure-related bleeding (Table 2). Interestingly, in all five patients with severe bleeding, it occurred shortly after PEG placement by the direct method; when the $24 \mathrm{~F}$ but- 
ton-bumper-type device was converted to the tube-bumper type device using the pull method, bleeding stopped rapidly. Therefore, the pull method may work more effectively than the direct method for the prevention of the PEG-related bleeding. The 27F dilator leaves a potential space between the $24 \mathrm{~F}$ button-bumper-type device and gastrostomy site. We speculated that this may prevent the device from compressing the wound edges and thus may not be hemostatic. When PEG is needed for inpatients in whom there are concerns related to possible bleeding from the PEG site, we prefer the standard pull method. The $27 \mathrm{~F}$ dilator allowed the easier placement of the device than when using $20 \mathrm{~F}$ or $24 \mathrm{~F}$ dilators but was associated with an increased risk of bleeding, making the use of the smaller diameter dilators a better choice clinically.

We concluded that instead of using the $27 \mathrm{~F}$ dilator contained in the commercial kit of the $24 \mathrm{~F}$ button-bumper-type device, using $20 \mathrm{~F}$ and 24F dilators during the PEG placement by the direct method may prevent procedure-related severe bleeding.

\section{References}

1. Horiuchi A, Nakayama Y, Tanaka N, et al. Prospective randomized trial comparing the direct method using a 24 Fr bumperbutton-type device with the pull method for percutaneous endoscopic gastrostomy. Endoscopy 2008;40:722-6.

2. Ferguson DR, Harig JM, Kozarek RA, et al. Placement of a feeding button ("one-step button") as the initial procedure. Am J Gastroenterol 1993;88:501-4.

3. Kozarek RA, Payne M, Barkin J, et al. Prospective multicenter evaluation of an initially placed button gastrostomy. Gastrointest Endosc 1995;41:105-8.

4. Maetani I, Tada T, Ukita T, et al. PEG with introducer or pull method: a prospective randomized comparison. Gastrointest Endosc 2003;57:837-41.

5. Horiuchi A, Nakayama Y, Kajiyama M, et al. Nasopharyngeal decolonization of methicillin-resistant Staphylococcus aureus can reduce peristomal wound infection. Am J Gastroenterol 2006;101: 274-7.

6. Brown MC. Cancer metastasis at percutaneous endoscopic gastrostomy stomata is related to the hematogenous or lymphatic spread of circulating tumor cells. Am J Gastroenterol 2000;95:3288-91.

7. Dormann AJ, Glosemeyer R, Leistner U, et al. Modified percutaneous endoscopic gastrostomy (PEG) with gastropexy: early experience with a new introducer technique. Z Gastroenterol 2000;38:933-8.

8. Dormann AJ, Wejda B, Kahl S, et al. Longterm results with a new introducer method with gastropexy for percutaneous endoscopic gastrostomy. Am J Gastroenterol 2006;101:1229-34.

9. Wejda BU, Deppe H, Huchzermeyer H, et al. PEG placement in patients with ascites: a new approach. Gastrointest Endosc 2005;61:178-80. 